\title{
TEMA -2018: Síndrome Compartimental, generalidades, consenso diagnóstico y técnica quirúrgica
}
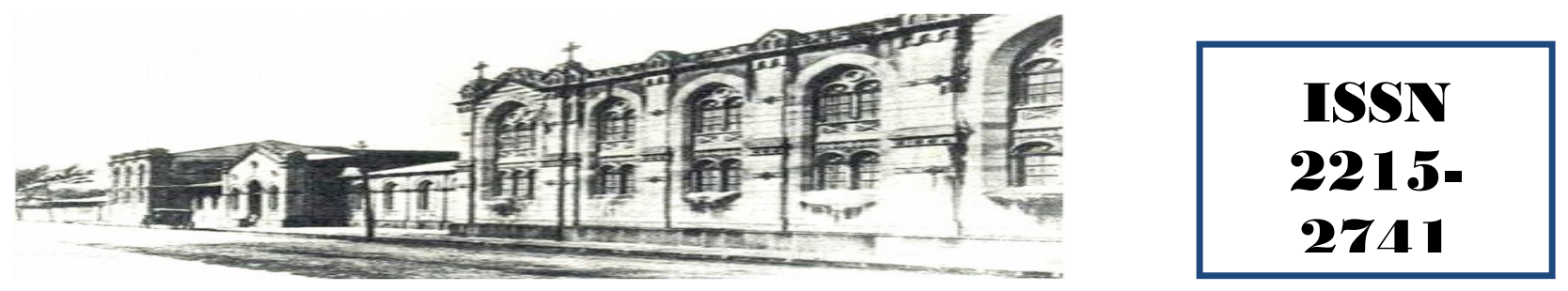

Haspital San quan de Dios, San José, Costa Rica. Fundado en 1845

Recibido:

$29 / 01 / 2018$

Aceptado:

$15 / 03 / 2018$

Adriana Paola Arroyo López ${ }^{1}$

Hebel Solano Múnoz ${ }^{2}$

Víctor Manuel Rojas Murillo ${ }^{3}$

${ }^{1}$ Médico general, Caja Costarricense del seguro social, Hospital de Alajuela, Costa Rica. adrial.arroyo@gmail.com

${ }^{2}$ Asistente en Cirugía General en el Hospital Max Peralta. hsmc1089@gmail.com

${ }^{3}$ Residente de cirugía general del Hospital Max Peralta. dr.victor.rojas@gmail.com

\section{RESUMEN}

El síndrome compartimental es una patología muy común, con una prevalencia mayor en el sexo masculino, asociada a traumatismos de fracturas expuestas, de tibia y antebrazo principalmente. Actualmente, existen muchas revisiones médicas, sin embargo, no se logra llegar a un consenso acerca del diagnóstico más certero de esta patología. Existen varios métodos diagnósticos que se encuentran en estudio y continua en duda si las mediciones intracompartimentales constituyen el método más preciso en el diagnóstico. Se presenta al lector una recopilación académica de los abordajes quirúrgicos empleados, métodos más actualizados de diagnóstico y generalidades acerca de esta emergencia quirúrgica.

\section{PALABRAS CLAVE}

Síndrome compartimental. Agudo, crónico y recurrente. Técnica de amarrado de cordones. Fasciotomía. Presiones intracompartimentales. Terapia de presión negativa. Técnica de fibra óptica.

\section{ABSTRACT}

Compartimental syndrome is a very common complication of motor vehicle accidents and compound fractures and it is considered a frequent emergency, specially if related to tibial and/or radial fractures. Currently there is no consensus for the diagnosis of this condition, however there is an astonishing amount of literature about diagnostic approaches and techniques, 
physical examination and anamnesis are the most common tools for diagnosis, but they're complemented by intracompartimental pressure measurement techniques. New procedures have been developed nevertheless their limitations are not clear due to the lack of clinical experience, regardless the focus of the treatment review is to present the most cutting edge and novel surgical interventions.

\section{KEY WORDS}

Comparment syndrome. Shoelace technique Fasciotomy. Negative pressure. Trauma. Non-ivasive techniques.

\section{INTRODUCCIÓN}

El síndrome compartimental representa una emergencia quirúrgica en todos los casos en los que se presenta. Es una patología sumamente común en sala de trauma y requiere un diagnóstico rápido, ya que el éxito del tratamiento radica en el abordaje breve con fasciotomía. Sin embargo, debido a la carencia de métodos diagnósticos, se introdujo la monitorización de la presión intracompartimental en $1970^{(1)}$.

La causa más frecuente del síndrome compartimental son las fracturas, representan un $69 \%$ de la totalidad de los $\operatorname{casos}^{(2)}$. Un $36 \%$ corresponden a fracturas de la diáfisis de la tibia, seguidas por las fracturas de radio distal en un $9.8 \%$ de los casos. Sin embargo, en los últimos estudios se ha demostrado que las fracturas mediales de rodilla han ido incrementando la incidencia de síndrome compartimental en al menos $53 \%{ }^{(1,2)}$.

\section{DISCUSIÓN}

\section{Etiologia}

El síndrome compartimental es una de las condiciones más frecuentes en los pacientes que presentan fracturas expuestas o fracturas por aplastamiento. En atletas es más común hallar el síndrome compartimental crónico ${ }^{(3)}$.

El síndrome compartimental agudo, se desarrolla por modificaciones entre el contenido de fluido y el tamaño del compartimento donde se encuentre la fractura o trauma; cualquier tipo de sangrado, ya sea por trauma vascular o sangrado proveniente del hueso esponjoso ${ }^{(3)}$, presenta una posibilidad de un $20 \%$ de desarrollarse en las extremidades que han sido revascularizadas ${ }^{(4)}$. Los vendajes circunferenciales, tales como los yesos pueden restringir la expansión del compartimento y ocasionar un aumento de la presión al igual que las prendas neumáticas antishock. También se puede presentar en quemaduras, rabdomiólisis, vasculitis autoinmunes, trombosis venosa profunda. La tracción y el reposicionamiento de una articulación ha mostrado afectar el volumen y la presión del compartimento. Muchos autores han hecho asociaciones al enclavado intramedular de las fracturas tibiales: el daño del tejido ocasiona edema. La tracción disminuye el volumen de los compartimentos, ocasionando obstrucción del flujo sanguíneo ${ }^{(1,2,3,4)}$.

Las infecciones son otra causa de síndrome compartimental, especialmente las causadas por Streptococcus, sin embargo el mecanismo aún no se ha determinado con precisión, esto se ha asociado a la exotoxina pirogénica de esta bacteria, cuya función de súper antígeno se cree que conduce a la lesión muscular directa ${ }^{(5)}$. En el caso del síndrome compartimental crónico, se presenta en los atletas que realizan movimientos repetitivos, como correr.

\section{Anatomia y fisiopatologia}

Tanto el síndrome compartimental agudo como el crónico, son el resultado del aumento de la presión intramuscular causada por eventos precipitantes.

\section{Funciones biomecánicas de la fascia ${ }^{(5)}$ :}

1. Proporcionar sitios de unión para los músculos.

2. Mantener la posición de los músculos durante el movimiento.

3. Mejorar la ventaja mecánica

del músculo durante el movimiento.

La fascia profunda por lo general se encuentra inervada, lo que probablemente juegue un papel importante en la coordinación y la propiocepción.

a. FUNDAMENTOS ANATOMICOS 
Los compartimentos son grupos musculares que se encuentran recubiertos por fascia inelástica, está fascia inelástica tiene la función de: mantener la forma de los tejidos y de brindar protección. Por las diferencias anatómicas que se presentan entre los adolescentes y adultos, es más común que el síndrome compartimental se desarrolle en adolescentes. Shadgan y colegas, establecieron que por lo general los adolescentes presentan una fascia mucho más fuerte, esto por el extenso relleno muscular. Aunado a esto, la práctica de actividades de alto impacto durante la adolescencia llegan a causar lesiones traumáticas que incrementan el riesgo ${ }^{(3,5)}$.

\section{Extremidad superior:}

Tiene dos compartimentos. El compartimento anterior contiene el bíceps y los músculos braquiales, además, el nervio ulnar, mediano y radial; el compartimento posterior contiene el tríceps.

En antebrazo encontramos dos compartimentos: el compartimento anterior contiene la muñeca y los extensores de los dedos; el compartimento posterior contiene los músculos flexores de la mano y dedos. La mano presenta 10 compartimentos:

- Interóseo dorsal: 4 compartimentos.

- Interóseo palmar: 3 compartimentos.

- $\quad$ Aductor del primer dedo, región tenar e hipotenar.

\section{Extremidad Inferior:}

En el muslo hay tres compartimentos: anterior (constituido por los músculos: vastos lateral, intermedio y lateral; sartorio y recto femoral), medial (constituido por los músculos: aductor largo, aductor corto y aductor mayor, además, el grácil) y posterior (constituido por los músculos semimembranoso, semitendinoso, bíceps femoral y el nervio ciático).

En la pierna hay 4 compartimentos que contienen estructuras musculares, arteriales y nerviosas. En el compartimento anterior las estructuras musculares: M. Tibial anterior. M. extensor de los ortejos; contiene estructuras vasculares y nerviosas: arteria tibial anterior y nervio peroneo profundo. El compartimento lateral con estructuras musculares, músculos: peroneo largo y corto, y estruc- turas nerviosas nervio peroneo superficial. El compartimento posterior se divide en: profundo con los músculos tibial posterior, flexor largo de los ortejos y flexor largo del hallux, además contiene la arteria tibial posterior y el nervio tibial posterior; el compartimento posterior superficial está constituido por los músculos gastronemio y sural y el nervio sural.

En el pie, el número actual de compartimentos y el tratamiento en el síndrome compartimental es controversial. Tres compartimentos fueron descritos inicialmente: medial, lateral y superficial. Actualmente se habla que el pie tiene 9 compartimentos: medial, lateral, cuatro interóseo y uno central $^{(5)}$.

\section{b. FUNDAMENTOS FISIOPATOLOGI- COS}

El tono vascular, la presión sanguínea, la duración de la elevación de la presión y las demandas metabólicas van a representar parámetros fundamentales en el desarrollo del síndrome compartimental agudo.

La vasodilatación precapilar en el sistema arteriolar, en conjunto con las vénulas colapsadas, aumenta la permeabilidad capilar, incrementando la tasa de filtración y la presión del líquido intersticial. La presión normal del líquido intersticial es de $10 \mathrm{mmHg}$, conforme está aumenta, la perfusión de los tejidos va descendiendo. Una vez que la perfusión de los tejidos alcanza niveles críticos, estos tejidos entran en una fase de hipoxemia.

La hipoxia incrementa el estrés oxidativo y se desarrolla hipoglicemia en el tejido, causado por el edema de las células, esto secundado al cierre de las bombas de sodio-potasio ATPasa, que mantienen el equilibrio osmótico de las células. La consiguiente pérdida de la membrana celular resulta en un influjo de iones de cloro que culmina en inflamación y necrosis celular. La isquemia muscular que se produce ocasiona la liberación de mioglobina, que es propiamente liberada en la circulación, con otros metabolitos inflamatorios y tóxicos. La mioglubinuria, la acidosis metabólica y la hipercalemia que se desarrolla, puede conducir a un fallo renal, shock, hipotermia y fallas o arritmias cardíacas ${ }^{(2,5)}$. 
El aumento de la presión va a depender del flujo sanguíneo local, que a su vez es determinado por la presión arterial, la presión venosa y la resistencia local vascular.

La presión de los tejidos va a ser proporcional a la diferencia entre la presión de perfusión y la presión del líquido intersticial, que se calcula utilizando la siguiente fórmula ${ }^{(6)}$ :

\section{Flujo sanguíneo local:}

(presión arterial local - presión venosa)

resistencia vascular local2

La isquemia del músculo esquelético ocasiona la liberación de sustancias similares a histamina, ocasionando el aumento de la permeabilidad vascular, secundando un espesamiento de la sangre con empeoramiento de la situación isquémica.

Las fracturas diafisiarias de la tibia y las fracturas distales de radio, son los factores de riesgo más frecuentes en el desarrollo de síndrome compartimental. El compartimento anterior de la pierna y el compartimento flexor del antebrazo son los más afectados ${ }^{(7)}$.

El síndrome compartimental crónico es una condición que induce dolor causado por el ejercicio, inflamación y alteración de la función muscular. En el caso de los síndromes compartimentales crónicos bilaterales, pueden ocurrir por una fascia adyacente insuficiente o por la hipertrofia muscular inducida por el ejercicio, lo que puede aumentar la masa muscular en un $20 \%$ dentro del espacio no elástico confinado ${ }^{(8)}$.

La inflamación muscular secundario a las actividades físicas repetitivas y a la estrechez de la fascia, son factores que se ven involucrados en el incremento de la presión del compartimento. Usualmente el dolor desaparece con reposo, pero el músculo afectado puede volverse isquémico ${ }^{(9)}$.

La isquemia del músculo esquelético ocasiona la liberación de sustancias similares a histamina, ocasionando el aumento de la permeabilidad vascular, secundando un espesamiento de la sangre con empeoramiento de la situación isquémica.
Las fracturas diafisiarias de la tibia y las fracturas distales de radio, son los factores de riesgo más frecuentes en el desarrollo de síndrome compartimental. El compartimento anterior de la pierna y el compartimento flexor del antebrazo son los más afectados ${ }^{(7)}$.

El síndrome compartimental crónico es una condición que induce dolor causado por el ejercicio, inflamación y alteración de la función muscular. En el caso de los síndromes compartimentales crónicos bilaterales, pueden ocurrir por una fascia adyacente insuficiente o por la hipertrofia muscular inducida por el ejercicio, lo que puede aumentar la masa muscular en un $20 \%$ dentro del espacio no elástico confinado ${ }^{(8)}$.

La inflamación muscular secundario a las actividades físicas repetitivas y a la estrechez de la fascia, son factores que se ven involucrados en el incremento de la presión del compartimento. Usualmente el dolor desaparece con reposo, pero el músculo afectado puede volverse isquémico ${ }^{(9)}$.

$\mathrm{Al}$ igual que en el síndrome compartimental agudo, en el síndrome compartimental crónico, el compartimento más afectado es el compartimento anterior de la pierna, además del compartimento posterior profundo. Ocurre bilateralmente entre el 37 al $82 \%$ de los atletas. Los esteroides anabólicos y los ejercicios excéntricos inducen la hipertrofia muscular, incrementando la presión intracompartimental y disminuyendo la elasticidad, predisponiendo a desarrollar el síndrome compartimental crónico ${ }^{(8,9)}$.

Otra potencial causa son las cicatrices miofasciales, la hipertensión venosa y el daño postraumático de los tejidos blandos, que lleva a inflamación.

\section{Clínica}

La ausencia de pulsos y un llenado capilar lento por lo general está relacionado con lesiones vasculares más que con síndrome compartimental.

Múltiples estudios han mostrado que la ausencia de síntomas de síndrome compartimental agudo, más que su presencia, favorecen al diagnóstico del mismo. Los síntomas y signos más frecuentes del síndrome compartimental son: dolor, parestesia y pérdida funcional 
de la extremidad comprometida. Los primeros signos clínicos aparecen con una presión intracompartimental entre $20-30 \mathrm{mmHg}$, la presión crítica es> $30 \mathrm{mmHg}^{(10)}$.

Se utiliza la nemotecnia de las cinco P en inglés.

Tabla 1. Clínica del Síndrome compartimental

\section{Pain: dolor.}

\section{Pallor: palidez.}

3. Pulselessness: ausencia de pulso.

4. Paresthesias: Parestesias.

5. Paralysis: Parálisis.

Fuente: Propia

Dentro de los signos y síntomas para el diagnóstico temprano cabe citar un estudio donde desarrollaron una tabla clínica con parámetros importantes para determinar la presencia temprana de síndrome compartimental en pacientes de trauma. Este estudio determina que es fundamental llevar a cabo un examen físico detallado, porque en algunos casos, el desarrollo de los síntomas se presenta hasta etapas tardías de la enfermedad ${ }^{(11)}$.

El 95\% de los niños que desarrollan síndrome compartimental agudo, han sufrido una fractura. Por lo general las alteraciones que se presentan en los niños están relacionadas con el trauma y no con el desarrollo de síndrome compartimental agudo, esto porque los infantes tienen un índice de tolerancia mayor en cuanto al tiempo de desarrollo de síndrome compartimental agudo ${ }^{(12)}$.

\section{Diagnostico}

Quizás uno de los retos más significativos del síndrome compartimental agudo, se produce en los pacientes que no pueden dar una historia clínica fiable o que no pueden participar en el examen físico. Esto incluye por ejemplo niños, pacientes con daño neurológico o pacientes politraumatizados. En esta clase de pacientes se debe llevar a cabo la medición de las presiones intracompartimentales.
El diagnóstico del síndrome compartimental crónico, puede ser más complicado, ya que solo se presenta síntomas con el aumento de la actividad física, y el diagnóstico va a depender de que tan rápido se mida la presión intramuscular una vez finalizado el ejercicio. Es importante tener un índice alto de sospecha y realizar exámenes físicos seriados. Se debe documentar el mecanismo de trauma del paciente. La ausencia del dolor en el síndrome compartimental es ocasionada por el déficit neurológico central o periférico. Los pulsos periféricos, son palpables a menos que haya un compromiso arterial masivo, rara vez, la presión del compartimento se eleva tanto que ocluye la presión arterial.

Las paresias pueden ser ocasionadas por isquemia muscular o la isquemia del nervio. La parestesia es un signo temprano de síndrome compartimental, que, sin tratamiento, progresa a hipoestesias y anestesias. Parálisis es un signo tardío del síndrome compartimental, que puede ser ocasionado por la compresión del nervio o lesión irreversible del músculo. Los síntomas sensoriales son frecuente indicación de isquemia del nervio. El tejido nervioso periférico, por lo general suele dañarse en mayor frecuencia, por tener mayor sensibilidad a los eventos isquémicos. La duración y el grado de presión que lleva a daño irreversible no está bien definido ${ }^{(2,6,13)}$.

La medición dentro del compartimento está indicada en las siguientes situaciones:

Tabla 2. Indicaciones para la medición dentro del compartimento.

\begin{tabular}{|l|l|l|}
\hline $\begin{array}{l}\text { Hipoten- } \\
\text { sión pro- } \\
\text { longada y } \\
\text { una ex- } \\
\text { tremidad } \\
\text { hinchada } \\
\text { con } \\
\text { firmeza. }\end{array}$ & $\begin{array}{l}\text { Uno o más } \\
\text { síntomas del } \\
\text { síndrome de } \\
\text { comparti- } \\
\text { mento con } \\
\text { factores de } \\
\text { confusión: } \\
\text { lesión neu- } \\
\text { rológica, la } \\
\text { anestesia } \\
\text { regional. }\end{array}$ & $\begin{array}{l}\text { Examen físico } \\
\text { poco fiable o im- } \\
\text { posible de } \\
\text { obtener con la } \\
\text { presencia de } \\
\text { firmeza o edema } \\
\text { en la extremidad } \\
\text { lesionada. }\end{array}$ \\
$\begin{array}{ll}\text { Aumento } \\
\text { espontáneo } \\
\text { de } \\
\text { dolor en }\end{array}$ & $\begin{array}{l}\text { Ningún otro sín- } \\
\text { la } \\
\text { extremidad } \\
\text { después }\end{array}$ & $\begin{array}{l}\text { toma } \\
\text { además de: au- } \\
\text { mento } \\
\text { de la firmeza o } \\
\text { edema } \\
\text { en la extremidad de } \\
\text { un }\end{array}$ \\
\hline
\end{tabular}




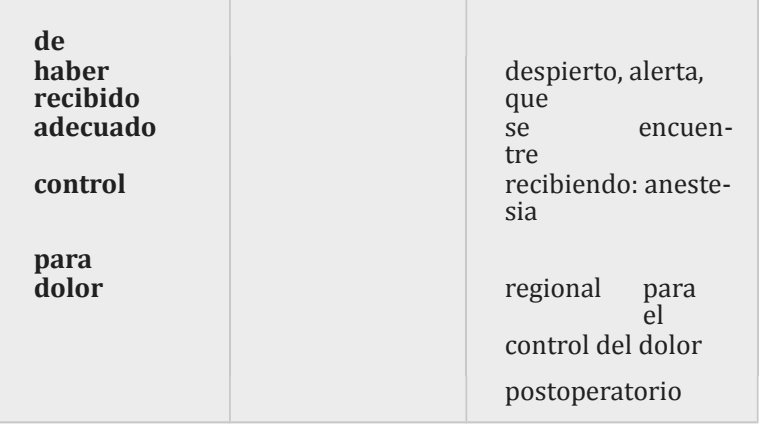

Fuente: Steven A. Olson, Robert R. Glasgow. Acute Compartment Syndrome in Lower Extremity Musculoskeletal Trauma. J Am Acad Orthop Surg 2005;13:436 444.

Las presiones intracompartimentales normales en adulto son de $8 \mathrm{mmHg}$. Tradicionalmente el diagnóstico de síndrome compartimental se ha llevado a cabo a través de la historia clínica y se ha complementado con la medición de las presiones. El límite de la presión intracompartimental ha sido debatido a lo largo del tiempo. Los expertos han abocado entre un límite máximo de $30-45 \mathrm{mmHg}$. Sin embargo la contraparte plantea que este límite es un poco extremo, y que los pacientes son sometidos innecesariamente a una fasciotomía ${ }^{(5,13)}$.

El uso de un valor definitivo para realizar una fasciotomía se ha ido cuestionando, porque la presión de perfusión necesaria para la oxigenación, es dependiente de la presión sanguínea del paciente. Algunos investigadores han propuesto el uso de una presión diferencial $(5,13)$ :

\section{$\Delta p=$ presión diastólica-presión intra- compartimental.}

Esto corresponde a un estudio, donde sometieron a estudio 116 pacientes con fracturas de diáfisis de tibia, valorando el uso continuo de la medición de la presión intracompartimental y encontraron que, de forma absoluta, la presión fue mayor a $30 \mathrm{mmHg}$ en el $46 \%$ de los pacientes; más de $40 \mathrm{mmHg}$ en el $26 \%$ de los pacientes y más de $50 \mathrm{mmHg}$ en el $3 \%$ de los pacientes $(5,13)$. Además un estudio prospectivo demostró que de 101 pacientes, la $\Delta \mathrm{p}$ tiene mayor valor diagnóstico que la presión intracompartimental por si sola.
Por lo general las presiones intracompartimentales en el miembro inferior en niños suele ser entre los 13 y los $16 \mathrm{mmHg}$, mucho mayor que los niveles que se presentan en los adultos esto porque el músculo se encuentra, en un período continuo de crecimiento y secundario a esto hay un aumento del volumen, por la hipertrofia, que suele presionar la fascia circundante $^{(14)}$.

Existen varias técnicas que se encuentran en estudio para realizar mediciones intracompartimentales, sin embargo, la eficacia de esos métodos todavía queda en duda. Los métodos para la detección del aumento de la presión intramuscular se pueden dividir en: sistemas de llenado de líquido que incluyen el complejo aguja/inyección, catéter de mecha y catéter de hendidura y los dispositivos de inyección de aguja Stryker. Los catéteres con punta de transductor incluyen: el transductor intracompartimental en estado sólido (STIC), además, el catéter de fibra óptica con punta de transductor ${ }^{(15)}$.

El método de llenado, requiere la infusión o pequeñas inyecciones de solución salina para mantener el catéter. También necesita ser calibrada y ajustada con respecto a la punta del catéter, esto en relación con el transductor extracorpóreo. Los transductores de fibra óptica son sistemas que no requieren el empleo de fluidos y tienen buenas propiedades dinámicas.

En estudios en animales se comprobó su utilidad, debido a su sensor de detección, en la medición de la presión intracompartimental en un músculo que se encuentre en reposo, bien hidratado y de igual forma en uno con presiones intracompartimentales elevadas. Se encuentra también la espectroscopia infrarroja; técnica nueva, no invasiva, que se basa en la absorción de luz cerca de un espectro infrarrojo, que corresponde a hemoglobina oxigenada y desoxigenada. La evaluación del tejido oxigenado fue realizada a través de una comparación con la oxihemoglobina y la desoxihemoglobina, concentradas en la sangre venosa, demostrando una relación inversa del compartimento, entre la presión y la oxigenación del compartimento en un modelo animal ${ }^{3}$, 15).

Los sistemas de medición deben ser fáciles de calibrar, de manejar y costo efectivos. La técnica 
de la fibra óptica no necesita ser recalibrada durante las mediciones y puede ser configurada para grabar en presión relativa o absoluta, utilizando unidades como $\mathrm{Pa}, \mathrm{mmHg}$, psi, bar y cmH2O. El método de aguja/inyección Stryker, es fácil de utilizar con un mínimo de instrucciones y es más costo efectivo. El proceso de calibración es rápido y fácil, sin embargo, la colocación del transductor externo en relación a la punta de la aguja, no debe cambiar luego de la calibración. Si no se cuenta con equipo sofisticado, la presión del compartimento se puede medir usando un tubo de administración intravenosa, una llave de paso de tres vías, una jeringa y un manómetro de mercurio. $(3,15)$

Un estudio comparativo entre la técnica del transductor sólido con catéter, el transductor electrónico de catéter de punta y la técnica de manómetro arterial lineal determinó que los tres métodos de medición no son fiables para realizar las medidas de tomas de presión de los compartimentos.(16)

Otro de los métodos utilizados son el ultrasonido de pulso con bucle de enganche, este dispositivo transmite un pulso acústico a modo de ráfaga a través del transductor de ultrasonido, el cual se encuentra acoplado a la superficie de la piel. Este se propaga a través de la piel y el tejido muscular $y$ se refleja en tejidos subyacentes, al mismo transductor que recibe la señal. Está técnica es capaz de detectar las formas de las ondas de desplazamiento, correspondientes a la pulsación arterial. Las ondas tienen formas características, por ejemplo, en el caso de la pierna, cuando la presión intracompartimental se encuentra elevada las formas de las ondas se vuelven menos complejas a medida que aumenta la presión dentro del compartimento. ${ }^{(17)}$

Además de ello existen otras técnicas que se han ido implementado por la carencia real de un parámetro de oro para hacer el diagnóstico. Dentro de los exámenes de sangre que se pueden realizar esta: la creatina fosfoquinasa, que refleja necrosis muscular y puede ser utilizado como un indicador de síndrome compartimental. En el caso de mioglubinuria refleja lisis celular. Este parámetro puede confundirse con hematuria, el diagnóstico definitivo se realiza con una prueba de bencidina en orina positiva, para sangre oculta en ausencia de glóbulos rojos.
Después del daño al músculo esquelético las concentraciones de mioglobina y la proteína fijadora de ácidos grasos se elevan significantemente, sin embargo la concentración de la creatina quinasa alcanza su máximo hasta dos horas después y se mantiene elevada por $48 \mathrm{~h}$. Se ha planteado que valores de 2000unidades/L después de una cirugía en pacientes ventilados y sedados son señales de advertencia de síndrome compartimental agudo.

Resonancia magnética nuclear (RMN): Se utiliza para detectar alteraciones de tejido blando y edema de los compartimentos. Sin embargo, la RMN no puede diferenciar el edema de los músculos afectados en un síndrome compartimental del edema de las lesiones de los tejidos blandos posterior a un trauma.

Flujometría con doppler/laser: Es una técnica bien desarrollada que mide la presión de perfusión de los tejidos. Está basado en el efecto doppler, que describe el cambio de la frecuencia de un sonido o bien la onda de luz, cuando la fuente de la onda y/o el receptor está moviéndose. Este método trabaja iluminando el tejido con un láser de bajo poder, basado en la circulación de células sanguíneas, mientras otra fibra óptica recoge la luz retrodispersada.

Además de este método, se han empleado otras formas para medir perfusión en tejidos. Hay diferentes marcadores bioquímicos que sirven para determinar hipoxia muscular: glucosa, lactato y niveles de piruvato pueden detectar isquemia en situaciones de oclusión muscular, hipertensión venosa e hipoperfusión. Se han realizado estudios recientes que han mostrado una mayor concentración de glucosa, en presencia de isquemia por oclusión de los vasos. ${ }^{(2,13,18)}$

La $\Delta \mathrm{P}$ es el parámetro más importante para determinar el diagnóstico más aún que la toma de las presiones compartimentales ${ }^{(19)}$.

\section{Manejo quirúrgico}


Figura 1. Sección transversal de la pierna de la demostración de los 4 compartimentos musculares anatómicos en miembro inferior.

Fuente: Reproducido de Amna Diwan, MD. Lower Leg Fasciotomy: 2-incision Technique. Techniques in Orthopaedics. Volume 27, Number 1, p 53-54, 2012.

Sin duda alguna, el síndrome compartimental, representa una emergencia quirúrgica, que debe ser diagnosticada con la mayor prontitud posible para evitar las secuelas en los pacientes. El abordaje quirúrgico empleado, la fasciotomía, con el fin de liberar a los músculos del aumento de la presión. Además, ya que el cierre inmediato suele estar contraindicado, se discutirán técnicas de cierre como el cierre tardío primario, la técnica de amarrado de cordones, (en inglés shoelace technique) y las técnicas de cierre con presión negativa.

La fasciotomía es preferible evitarla cuando la presión de perfusión dentro del compartimento no sea mayor de $30 \mathrm{mmHg}$. Dentro de las contraindicaciones se encuentran: coagulopatías que representan una causa de exanguinación en el paciente. Una contraindicación relativa es diagnóstico tardío de síndrome compartimental cuando la necrosis muscular ya está presente. Dentro de las consecuencias de una fasciotomía se encuentran: problemas sensitivos, herniación muscular, cicatrices queloides y ulceraciones recurrentes. En los estudios realizados se evidenció que en los pacientes en los que se retrasó la fasciotomía a un tiempo mayor de $12 \mathrm{~h}$ las tasas de amputación rondan entre los $3.2 \%$ y $14 \%$.

Entre 6 y $12 \mathrm{~h}$ las tasas de mortalidad van de un 2 a un $4.3 \%$ (19). Un alto índice de sospecha se debe de mantener en los pacientes pediátricos con lesiones traumáticas ${ }^{(20)}$.

La recurrencia de un síndrome compartimental luego de una fasciotomía, suele ser raro, los casos que han sido publicados están relacionados con: desórdenes de tejido conectivo, miositosis viral y trauma. Se documentó un caso en la literatura en relación a la reperfusión después del período de isquemia ${ }^{(21)}$.
Fasciotomía Miembro superior: Para descompri-

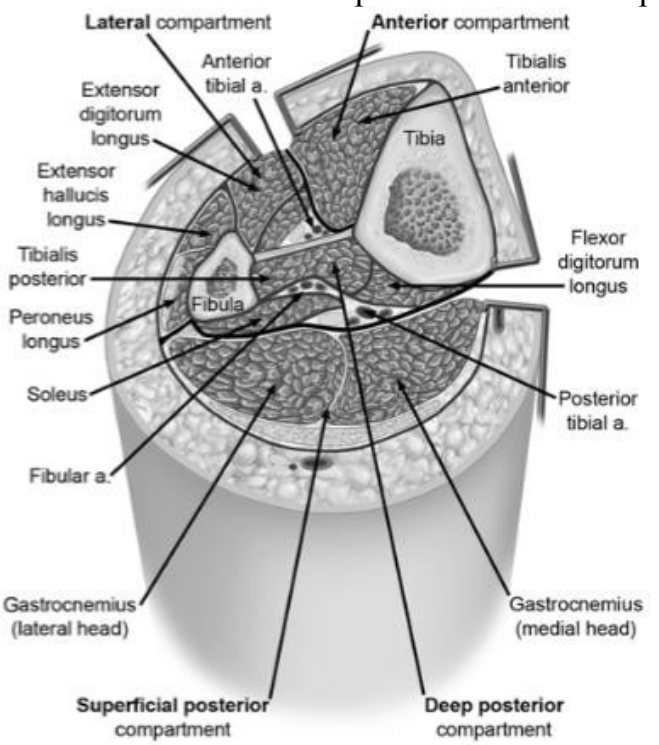

mir el brazo, se debe realizar una incisión anterior a lo largo del bíceps. La fascia del bíceps y los músculos braquiales que se encuentran por debajo, son liberados fácilmente. Si es necesario el tríceps es descompresionado con una incisión posterior. Si se necesita, la incisión anterior se puede extender a lo largo del codo y puede ser incorporada en una fasciotomía anterior del brazo. En el codo la incisión debe realizarse a través de su pliegue flexor en forma de zigzag a fin de evitar una contractura más tarde. La incisión es continuada distalmente en el antebrazo tal como sea necesario. Para las incisiones profundas del antebrazo, el abordaje es entre: el flexor carpo ulnar y el flexor digital superficial. Es necesario dividir una o dos ramas de la arteria ulnar, distal al flexor digital superficial, en función de exponer el pronador cuadrado.

En la muñeca se realiza un procedimiento idéntico a la liberación del nervio mediano en el túnel carpal. La contractura isquémica de Volkman es una compleja y variable deformidad en flexión de la muñeca y de los dedos, resultado de la fibrosis y la contractura de los músculos flexores del antebrazo, por la lesión isquémica de los tejidos profundos, encerrando de manera estrecha los compartimentos osteofasciales secundarios a un síndrome compartimental. Está asociado en algunas ocasiones con la mala unión o no unión del antebrazo ${ }^{(5,22)}$.

\section{Región Glútea, Muslo y Pie:}




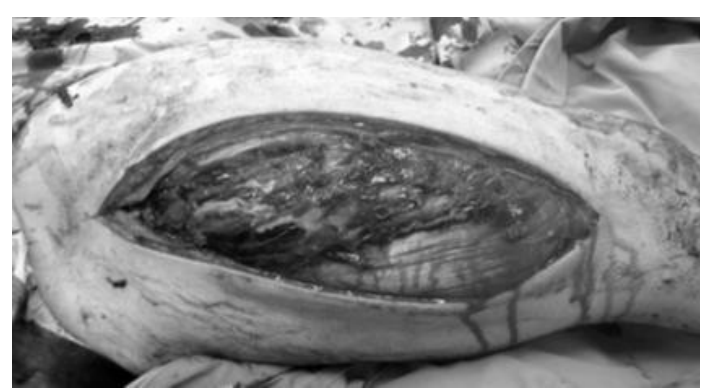

Figura 2. Fasciotomía lateral del muslo. La banda iliotibial también tiene una incisión así como la fascia del vasto lateral.

Fuente: Andrew H. Schmidt, MD. Acute. Compartment Syndrome. Orthop Clin N Am 47 (2016) 517-525.

En región glútea se realiza una incisión posterior que permite tener acceso al glúteo mayor. Para realizar la descompresión del pie generalmente se utilizan dos incisiones en el dorso del pie, una sobre el centro del 4 metatarsiano y otra sobre el espacio entre el primero y segundo metatarsiano. Expertos aclaran que la morbilidad de la fasciotomía plantar excede las secuelas del síndrome compartimental per se, pudiendo ocasionar la claudicación en los ortejos. El pie tiene numerosos compartimentos funcionales, sin embargo, el aductor del pulgar, los músculos interóseos y el cuadrado plantar, son valiosos en las fasciotomías plantares; una adecuada descompresión requiere la liberación de la fascia y de los músculos intrínsecos adheridos al pie. La contractura isquémica del cuadrado plantar secundario a las presiones dentro del compartimento, pueden causar deformidades, especialmente en fracturas del calcáneo.

En el caso del muslo se realiza una incisión única lateral para descomprimir los compartimentos anterior y posterior. En algunas ocasiones la incisión del aductor medial es requerida. El paciente es colocado en posición supina con un poco de elevación a nivel ipsilateral en cadera. Para liberar los compartimentos anterior y posterior se dibuja una línea desde la punta del trocánter mayor, proximal al epicóndilo lateral del fémur distal, se realiza una incisión, de la piel y los tejidos, proximal a la fascia lata y la banda iliotibial.

A nivel de la fascia lata/banda iliotibial, exponiendo el vasto lateral, es elevado fuera del septo intermuscular y retraído anteromedialmente; exponiendo de esta manera el septo lateral intermuscular. El compartimento posterior es liberado a través de la incisión en el compartimento anterior, mediante a incisión en el septo intermuscular, lateral y longitudinal a incisión, a 3-5 cm de la línea áspera ${ }^{(1,5,23)}$.

Si la presión del compartimento medial se encuentra dentro de la presión diastólica preoperatoria, (P $\Delta 30 \mathrm{mmHg}$ ), luego de la descomprensión de los compartimentos lateral y posterior, se debe realizar una fasciotomía del compartimento medial. Para esto se elimina la elevación ipsilateral que se aplicó antes de iniciar la cirugía. Y la cadera es flexionada y abducida, se coloca en rotación externa. El tubérculo del pubis y el hiato safeno son palpados y la incisión inicia entre estos dos, siguiendo el curso hacia el epicóndilo medial a lo largo del curso de la vena safena. El septo intermuscular medial es aproximado lateral y anterior al músculo aductor largo. Se realiza una incisión entre el grácil y el aductor largo, para inspeccionar el aductor corto y mayor.

\section{Pierna:}

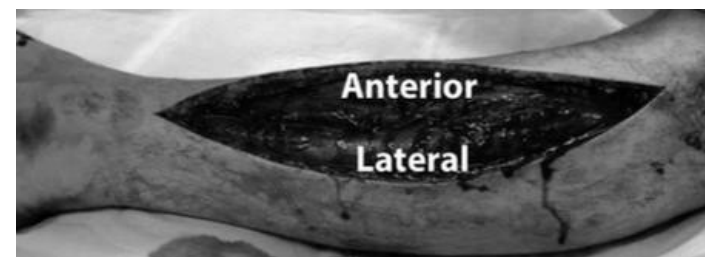

Figura 3. Fasciotomía lateral de la pierna mostrando compartimentos anterior y lateral.

Fuente: Andrew H. Schmidt, MD. Acute. Compartment Syndrome. Orthop Clin N Am 47 (2016) 517-525.

En las fracturas de tibia, la incidencia de síndrome compartimental va de 1 a $53 \%$, con las mayores incidencias reportadas en fracturas asociadas en dislocación de rodilla (53\%), y la menor incidencia en fracturas de tercio distal de la tibia $(1 \%),{ }^{(24)}$. 
Se pueden llevar a cabo dos incisiones una doble o bien una fasciotomía con una única incisión. Incisión Doble: Una incisión medial es realizada para liberar los compartimentos posteriores. Para poderla realizar se deberán palpar el borde subcutáneo posteromedial de la tibia, se lleva a cabo una incisión de $1.5 \mathrm{a} 2 \mathrm{~cm}$ posterior a este punto. La incisión se debe extender a lo largo de la tibia, desde aproximadamente el tubérculo tibial a la unión musculotendinosa del tendón de Aquiles.

Se debe diseccionar hasta la fascia, una vez que está es identificada (fascia), se llevan a cabo dos incisiones. La primera incisión se hace $1 \mathrm{~cm}$ posterior al borde posteromedial de la tibia, una vez realizada la incisión con unas tijeras de Metzenbaum se corta a lo largo de la fascia, extendiéndose proximal y distalmente, paralelo al eje de la tibia, esto se realiza a lo largo de la incisión que se realizó en piel, liberando así el compartimento posterior profundo. La incisión anterolateral deberá ser llevada a cabo para liberar los compartimentos anterior y lateral.

Se debe palpar el eje fibular y la cresta anterior de la tibia. Se hace una incisión aproximadamente $2 \mathrm{~cm}$ anterior al eje de la fíbula, la extensión de la incisión debe extenderse a lo largo de la longitud de la pierna, $4 \mathrm{~cm}$ por debajo de la cabeza de la fíbula hasta la unión miotendinosa de los peroneos. Se realiza una incisión en el compartimento anterior y con las tijeras de Metzenbaum se completa la fasciotomía, extendiendo la fasciotomía a lo largo de la incisión en piel. El nervio peroneo superficial debe ser identificado antes de descomprimir el compartimento, Una vez que el nervio es identificado, se realiza otra incisión en la fascia del compartimento lateral, posterior al nervio y con las tijeras de Metzenbaum nuevamente son utilizadas e insertadas para extender la incisión proximal y distalmente a lo largo de la longitud de la incisión en piel ${ }^{(25)}$.

\section{Tipos de cierre}

El cierre primario en la teoría, resulta más funcional y estético, disminuyendo de forma considerable la morbilidad del paciente, pero no es factible, debido al edema muscular que se presenta, la protrusión del músculo a través de la fascia y la significante retracción de la piel y ciertamente no puede ser realizada durante el procedimiento inicial quirúrgico. Dos de las téc- nicas más empleadas para el cierre definitivo de las heridas por fasciotomías entran en el rango del cierre primario tardío y son: la técnica de amarrado de cordones, (en inglés, shoelace technique) y las técnicas de presión negativa ${ }^{(26) .}$

Terapia con presión negativa: La terapia presión negativa en las heridas, siglas en inglés NPWT (negative-pressure wound therapy), se basa en la necesidad de cubrir y drenar las heridas traumáticas de tejido blando y los defectos asociados con las fracturas expuestas ${ }^{(27)}$.

Componentes de la terapia presión negativa en las heridas ${ }^{(28)}$ :

- Esponja de poros abiertos: están compuestos por poliuretano o polivinilo de alcohol. Está estructura permite que la presión negativa sea transmitida a lo largo de la interfaz esponja que se encuentra cubriendo la herida.

- Vendaje semioclusivo: Se utilizan para sellar la herida y proveer una adecuada succión.

- Fuente de la presión negativa: Tiene una excelente respuesta en la formación de tejido de granulación.

Mecanismo de Acción:

1. Contracción de la herida.

2. Disminución del edema.

3. Remoción del exudado de la herida.

La meta en las heridas por fasciotomía es disminuir la presión dentro del compartimento, llevando consigo la mejora de la perfusión. El cierre primario en fasciotomías no puede ser realizado y está contraindicado. Comúnmente se utilizan técnicas posoperatorias que incluyen cambios reiterativos en los apósitos estériles. La idea de estos continuos cambios es permitir que la herida se mantenga limpia mientras la inflamación resuelve, previo al cierre definitivo o mientras se cubre con un injerto de piel de espesor parcial. Quizás una de las mayores desventajas de este tipo de herida, es la exposición de la misma a un ambiente hospitalario, por el riesgo elevado de contaminación de la herida ${ }^{(28)}$. Se puede emplear presión subatmosférica continua o intermitente (29). 
En varios estudios retrospectivos en donde se realizaron fasciotomías con cierre primario tardío o con injertos de piel, y se utilizó terapia de presión negativa, el cierre se logró realizar en 6 días (30). En un estudio que se realizó con animales (cerdos), a los cuales se les fue inducido un síndrome compartimental, para poder documentar la función de la terapia con presión negativa, se demostró que no disminuye el peso muscular del músculo, sin embargo quedó demostrado la disminución en la regeneración de la fibra muscular en el tejido en donde se utilizó el dispositivo de presión negativa, en comparación en los animales controles, donde se utilizaron únicamente gasas y apósitos ${ }^{(31)}$.

Técnica de amarrado de cordones: En esta técnica se utiliza un material que en inglés es conocido como silastic. Estas son sustancias poliméricas de silicón que tienen propiedades como el caucho. Se colocan grapas a una distancia de 1.5 a $2 \mathrm{~cm}$ en los márgenes de la herida, luego de esto, dos bandas de silastic, son introducidas a través de las grapas, para poder realizar un cierre, tal como se amarran los cordones y para gradualmente lograr el cierre de la herida ${ }^{(26,32)}$. Una vez que la piel se encuentra aproximada por completo, las gasas y el silastic son removidos y la epitelización de la herida se completa en un periodo de 2 a 3 días.

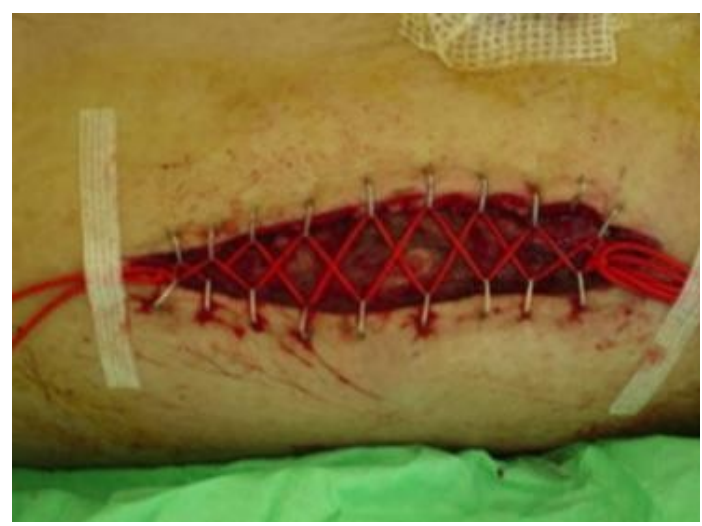

Figura 4. Aproximación con técnica de amarrado de cordones.
Fuente: Kakagia D1, Karadimas EJ2, Drosos G3, Ververidis A3, Trypsiannis G4, Verettas D3. Wound closure of leg fasciotomy: comparison of vacuum-assisted closure versus shoelace technique. A randomised study. Injury. 2014 May;45(5):890-3. doi: 10.1016/j.injury.2012.02.002.

\section{Técnicas alternativas en Síndrome Comparti- mental Crónico:}

El síndrome compartimental crónico es una condición que induce dolor causado por el ejercicio, inflamación y alteración de la función muscular. Ocurre bilateralmente entre un 37 al $82 \%$ de los atletas. Los esteroides anabólicos y los ejercicios excéntricos inducen la hipertrofia muscular, incrementando la presión intracompartimental $\mathrm{y}$ disminuyendo la elasticidad, predisponiendo a desarrollarlo ${ }^{(33)}$. En el caso de estos pacientes además de la optar por realizar una fasciotomía, hay estudios que plantean que mejorar la técnica al correr mejora los síntomas.

Los pacientes corredores representan un $69 \%$ de los pacientes con síndrome compartimental crónico. La técnica de corrido influencia las presiones en el compartimento anterior de la pierna. En concreto las presiones del compartimento anterior descienden cuando hay un patrón de contacto utiliza el ante pie cuando se está corriendo ${ }^{(34)}$.

\section{Prevención}

Remover yesos o vendajes compresivos puede ayudar a disminuir el riesgo d desarrollar síndrome compartimental. La elevación de la extremidad. Utilizar hielo en la extremidad afectada. Aplicar flujo de oxígeno en la pierna comprometida.

Utilizar vendaje compresivo en la extremidad afectada. También se establece que el paciente se debe mantener normotenso con flujo de oxígeno alto, con saturación óptima de oxígeno para optimizar la presión de perfusión y el oxígeno en el compartimento.

\section{CONCLUSIONES}

El síndrome compartimental representa una emergencia quirúrgica, que requiere un abordaje preciso y rápido para evitar los efectos adversos que ocasiona sobre la extremidad. A pesar de que existen muchos estudios con respecto al diagnóstico efectivo, hoy por hoy no existe un "gold es- 
tándar", o una medida de oro que pueda ser efectuada para determinar a ciencia cierta si el paciente presenta o no tal cuadro clínico. Se siguen empleando la historia clínica y examen físico, con la suplementación de las medidas de las presiones intracompartimentales.

\section{BIBLIOGRAFÍA}

1. Andrew H. Schmidt, MD. Acute. Compartment Syndrome. Orthop Clin N Am 47 (2016) 517-525. http://dx.doi.org/10.1016/j.ocl.2016.02.001

2. Steven A. Olson Robert R. Glasgow. Acute Compartment Syndrome in Lower Extremity Musculoskeletal Trauma. J Am Acad Orthop Surg 2005; 13:436- 444.

3. Hasnain Raza Anant Mahapatra, Acute Compartment Syndrome in Orthopedics: Causes, Diagnosis, and

Management, Advances in Orthopedics, vol. 2015, Article ID 543412, 8 pages,

2015. doi: $10.1155 / 2015 / 543412$

4. G. J. Mar M. J. Barrington B. R. McGuirk. Acute compartment syndrome of the lower limb and the effect of postoperative analgesia on diagnosis. Br. J. Anaesth. (2009) 102 (1): 3- 11.doi: 10.1093/bja/aen330

5. Arvind $\mathrm{G}$ von Keudell Michael $\mathrm{J}$ Weaver Paul T Appleton Donald S Bae George S M Dyer Marilyn Heng et al. Diagnosis and Treatment of acute extremity compartment sindrome. P1299-1310, 26 September 2015. doi.org/10.1016/S0140- 6736(15)00277-9

6. McLaughlin $\mathrm{N}$ Heard $\mathrm{H}$ Kelham $\mathrm{S}$. Acute and chronic compartment syndromes: know when to act fast. JAAPA. 2014 Jun;27(6):23-26. doi: 10.1097/01.JAA.0000446999.10176.13.

7. Parveen Ali Julie Santy-Tomlinson Roger Watson. Assessment and diagnosis of acute limb compartment syndrome: A literature review. International Journal of Orthopaedic and Trauma Nursing. November 2014 Volume 18, Issue 4, Pages 180-190 http://dx.doi.org/10.1016/j.ijotn.2014 .01 .002
8. Aweid O Del Buono A Malliaras P Iqbal $\mathrm{H}$ Morrissey D Maffulli N et al. Systematic review and recommendations for intracompartmental pressure monitoring in diagnosing chronic exertional compartment syndrome of the leg. Clin J Sport Med. 2012

10.1097/JSM.0b013e3182580e1d.

Jul;22(4):356-370.doi:

9. Nicola Maffull Mattia Loppini Filippo Spiezia Alessio D'Addona Gayle D. Maffulli. Single minimal incision fasciotomy for chronic exertional compartment syndrome of the lower leg. J Orthop Surg Res. 2016; 11: 61. doi: 10.1186/s13018-016-0395-9

10. T. Chandraprakasam R. Ashok Kumar. Acute compartment syndrome of forearm and hand. Indian J Plast Surg. 2011 May-Aug; 44(2): 212-218.

doi: $10.4103 / 0970-0358.85342$

11. Ferlic PW Singer G Kraus T Eberl R. The acute compartment syndrome following fractures of the lower leg in children. Injury. 2012 Oct;43(10):1743-1746. doi: 10.1016/j.injury.2012.06.025.

12. Kosir R Moore FA Selby JH Cocanour CS Kozar RA Gonzalez EA et al. Acute lower extremity compartment syndrome (ALECS) screening protocol in critically ill trauma patients. J

Trauma. 2007 Aug;63(2):268-275.

13. Babak Shadgan Matthew Menon David Sanders Gregg Berry Claude Martin, Jr. Paul Duffy, David Stephen et al. Current thinking about acute compartment syndrome of the lower extremity. Can J Surg. 2010 Oct; 53(5): 329-334. PMCID: PMC2947124

14. Erdös J Dlaska C Szatmary

$\mathrm{P}$ Humenberger M Vécsei V Hajdu S. Acute compartment syndrome in children: a case series in 24 patients and review of the literature. Int Orthop. 2011 Apr;35(4):569-575. doi: 10.1007/s00264-010-1016-6. Epub 2010 Apr 18.

15. Nilsson A Zhang Q Styf J. Evaluation of a fiber-optic technique for recording intramuscu- 
lar pressure in the human leg. J Clin Monit Comput. 2016 Oct;30(5):699-705. doi: 10.1007/s10877-015-9750-3. Epub 2015 Aug 14.

16. Collinge C Kuper M. Comparison of three methods for measuring intracompartment al pressure in injured limbs of trauma patients. $\mathrm{J}$ Orthop Trauma. 2010 Jun;24(6):364- 368. doi: 10.1097/BOT.0b013e3181cb5866.

17. John M. Wiemann Toshiaki Ueno Bryan T. Leek William T. Yost Alexandra K. Schwartz Alan R. Hargens. Noninvasive Measurements of Intramuscular Pressure Using Pulsed Phaselocked Loop Ultrasound for Detecting Compartment Syndromes. J Orthop Trauma 2006;20:458463

18. Doro CJ Sitzman TJ O'Toole RV. ¿Can intramuscular glucose levels diagnose compartment syndrome? J Trauma Acute Care Surg. 2014 Feb;76(2):474-

8. doi: 10.1097/TA.0b013e3182a9ccd1.

19. H Hayakawa DJ Aldington, RA Moore. Acute traumatic compartment syndrome: a systematic review of results of fasciotomy. Trauma 2009; 11: 5-35

20. Wallin K Nguyen H Russell L Lee DK. Acute Traumatic Compartment Syndrome in Pediatric Foot: A Systematic Review and Case Report. J Foot Ankle Surg. 2016 JulAug;55(4):817-820. doi: 10.1053/j.jfas.2016.02.010.

21. Ashwini P. Kerkar Alik Farber Jeffrey A. Kalish, Jeffrey J. Siracuse. Recurrent Lower-Extremity Compartment Syndrome after Four-Compartment Fasciotomy Secondary to Acute Limb Ischemia. Ann Vasc Surg. 2016 Jan;30:306.e5-7. doi: 10.1016/j.avsg.2015.06.092. Epub 2015 Sep 9.

22. Maheshwar L Kiran K K Vamshi KC Siva Prasad R. Volkmann's Ischemic Contracture with Atrophic Non-union of Ulna Managed by Bone Shortening and Transposition of Radial Autograft. Journal of Orthopaedic Case Reports 2015 Jan-March: 5(1):Page 65-68 doi:10.13107/jocr.2250-0685.259.
23. Jeffrey MacLean Rosanna Wustrack Utku Kandemir. Compartment Syndrome of Thigh. Tech Orthop 2012;27: 47-52

24. Michael J. Weaver Trevor M. Owen Jordan H. Morgan Mitchel B. Harris. Delayed Primary Closure of Fasciotomy Incisions in the Lower Leg: Do We Need to Change Our Strategy?. J Orthop Trauma. 2015 Jul;29(7):308-311. doi: 10.1097/BOT.0000000000000278.

25. Amna Diwan. Lower Leg Fasciotomy: 2- incision Technique. Techniques in Orthopaedics. Volume 27, Number 1, p 53-54, 2012. doi: 10.1097/BTO.0b013e318249aad4

26. Kakagia D Karadimas EJ Drosos G Ververidis A Trypsiannis $G$ Verettas D. Wound closure of leg fasciotomy: comparison of vacuum-assisted closure versus shoelace technique. A randomised study. Injury. 2014 May;45(5):890-893. 10.1016/j.injury.2012.02.002.

27. Streubel PN Stinner DJ Obremskey WT. Use of negative-pressure wound therapy in orthopaedic trauma. J Am Acad Orthop Surg. 2012 Sep;20(9):564-574. doi: 10.5435/JAAOS-20-09564.

28. Pollak AN. Use of negative pressure wound therapy with reticulated open cell foam for lower extremity trauma. J Orthop Trauma. 2008 Nov-Dec;22(10 Suppl):S142-5. doi: 10.1097

29. Stannard JP Volgas DA Stewart

$\mathrm{R}$ McGwin G Jr Alonso JE. Negative pressure wound therapy after severe open fractures: a prospective randomized study. J Orthop

Trauma. 2009 Sep;23(8):552-557. doi: 10.1097/BOT.0b013e3181a2e2b6.

30. Kanakaris NK Thanasas C Keramaris N Kontakis G Granick MS Giannoudis PV. The efficacy of negative pressure wound therapy in the management of lower extremity trauma: review of clinical evidence. Injury. 2007 Dec;38 Suppl 5:S9-18. Epub 2007 Nov 28.

31. Wilkin G Khogali S Garbedian S Slagel B Blais S Gofton W. Negative-pressure wound therapy after fasciotomy reduces muscle-fiber re- 
generation in a pig model. J Bone Joint Surg Am. 2014 Aug 20;96(16):1378-1385. doi: 10.2106/JBJS.M.01010.

32. Zorrilla P Marín A Gómez LA Salido JA. Shoelace technique for gradual closure of fasciotomy wounds. J Trauma. 2005 Dec;59(6):1515-1517.

33. Aweid O Del Buono A Malliaras P Iqbal H, Morrissey D Maffulli N Padhiar N. Systematic review and recommendations for intracompartmental pressure monitoring in diagnosing chronic

exertional compartment syndrome of the leg. Clin J Sport Med. 2012 Jul;22(4):356-370. doi: 10.1097/JSM.0b013e3182580e1d.

34. Diebal AR Gregory R Alitz C Gerber JP. Forefoot running improves pain and disability associated with chronic exertional compartment syndrome. Am J Sports Med. 2012 May;40(5):1060- $1067 . \quad$ doi: 10.1177/0363546512439182. Epub 2012 Mar 16.

\section{CONFLICTO DE INTERÉS Y/O AGRADECIMIENTOS}

Los autores declaran que no existió ningún conflicto de interés en el presente reporte. 